\title{
AN IN VITRO STUDY TO EVALUATE THE COLOR STABILITY OF FOUR DIFFERENT CAD/CAM CERAMICS
}

\author{
Rana M Sherif*
}

\begin{abstract}
Statement of the problem: Dental Ceramics have been widely used in patients seeking ultimate esthetics, however still there is concern about their color stability by time.
\end{abstract}

Aim of the study: To evaluate the color stability of four different CAD/CAM ceramics after immersion in coffee solution.

Materials and Methods: A total of twenty ceramic discs with shade A2 (10 mm in diameter and $2 \mathrm{~mm}$ in thickness) were constructed in standardized manner. Samples were divided into four main equal groups $(n=5)$ each, according to the type of ceramic material used. Group I: IPS e.max CAD. Group V: Vita Suprinity. Group C: Cerec Blocs. Group O: Obsidian. All specimens were fabricated using CNC milling machine and electric isoMet microsaw 4000. A specially constructed cylindrical split teflon mold was fabricated for the construction of twenty composite resin discs shade A3 (10 $\mathrm{mm}$ in diameter and $2 \mathrm{~mm}$ in thickness). Both ceramic and composite discs were constructed according to manufacturer instructions then were adhesively luted using dual cure Rely X U200 Automix self-Adhesive resin cement shade A2 under $2 \mathrm{Kg}$ constant load application. All disc samples were immersed in coffee solution for 3 weeks and were tested twice; before (baseline) and after the immersion. The color of each sample was then assessed using a spectrophotometer. The $L^{*} a^{*} b^{*}$ values of the samples were recorded according to the CIELAB color scale relative to the standard illumination D65. The results were tabulated and statistically analyzed as mean, standard deviation (SD), median, range and with $95 \%$ Confidence interval values. For parametric data, one-way ANOVA was used followed by Tukey's post-hoc test to compare between the four groups. While for non-parametric data, Kruskal-Wallis test was used to compare between the four groups. The significance level was set at $\mathrm{P} \leq 0.05$. Statistical analysis was performed with IBM® SPSS $®$ Statistics Version 20 for Windows.

Results: There was a statistically significant difference between the four ceramic groups after immersion in coffee solution. A decrease in $\left(\mathrm{L}^{*}\right)$ values was found in all groups. Pair-wise comparisons revealed that IPS e.max CAD showed the highest mean decrease in $(\Delta \mathrm{L})(-5.19 \pm 0.71)$.

* Assistant Professor, Fixed Prosthodontics Department, Faculty of Oral and Dental Medicine, Cairo University, Egypt. 
While no statistically significant difference was seen between mean $(\Delta \mathrm{L})$ of Cerec Blocs, Obsidian and Vita Suprinity respectively $(-2.06 \pm 1.17 ;-1.48 \pm 1.21 ;-2.04 \pm 0.31)$ all showed lower decrease in $\left(\mathrm{L}^{*}\right)$ value than IPS e.max CAD. For $\Delta \mathrm{b}$ a statistically significant difference was found between the four ceramic groups and pair-wise comparisons revealed that Obsidian showed the highest mean $(\Delta \mathrm{b})(5.30 \pm 0.15)$ with non-statistically significant difference from Vita Suprinity (4.77 $\pm 0.52)$. Cerec Blocs showed lower mean $(\Delta \mathrm{b})(4.34 \mathrm{~b} \pm 0.18)$ with non-statistically significant difference from Vita Suprinity but with a statistically significant difference from Obsidian. IPS e.max CAD showed the statistically significantly lowest mean $(\Delta \mathrm{b})(2.04 \pm 0.49)$. For $(\Delta \mathrm{a})$ and $(\Delta \mathrm{E})$ non-statistically significant difference was found among the four different ceramic groups (Cerec Blocs, Obsidian, Vita Suprinity ,IPS e.max CAD) respectively after immersion in coffee solution, where mean $(\Delta \mathrm{a})$ was $(3.07 \pm 0.12 ; 3.18 \pm 0.13 ; 2.93 \pm 0.18 ; 3.07 \pm 0.61)$ and mean $(\Delta \mathrm{E})$ was $(5.78 \pm 0.49 ; 6.44 \pm 0.40 ; 5.97 \pm 0.45 ; 6.39 \pm 0.88)$

Conclusions: The color stability of the four tested CAD/CAM ceramics was affected by immersion in coffee solution and they became darker in appearance.

KEY WORDS: dental ceramics, CAD/CAM, coffee solution, spectrophotometer, color stability.

\section{INTRODUCTION}

Metal-ceramic restorations have been always considered as the gold standard for restoring damaged teeth. They are favored for their mechanical and biological properties together with satisfactory esthetic results. However, the metal substructure is often visible as a thin, gray line at the crown margin, that does not transmit light in a similar way as natural teeth, and thus results in a dull appearance, especially in teeth with light cervical color $^{(1,2)}$. Therefore the increase in patient esthetic demands resulted in the creation of new number of dental ceramics which gained popularity due to their excellent esthetic, great biocompatibility, and good wear resistance ${ }^{(3)}$. Recently, metal-free restorations had undergone many improvements whether in the technique of fabrication or in the material itself to enhance the quality of the final restoration regarding color stability, surface texture, strength and marginal fit ${ }^{(4,5) \text {. }}$

The introduction of computer-aided designing computer-aided manufacturing (CAD/CAM) systems to restorative dentistry represent a major technological breakthrough with many advantages over the conventional one. The system uses industrial ceramic blocks which are prefabricated under optimum and controlled conditions to mill ceramic restorations and thus delivering a high quality restoration with improved mechanical and optical properties without the inevitable inaccuracies seen in the manually-produced one. ${ }^{(5-8)}$

Many types of CAD/CAM ceramic blocks have been developed for restoration fabrication, including feldspathic ceramic, leucite-reinforced ceramics, lithium disilicate ceramics, and zirconium oxide ceramics ${ }^{(9)}$. Feldspathic CAD/CAM ceramics are glass based ceramics with superior translucency, available in different shades but with a low flexural strength of $154 \pm 0.5 \mathrm{MPa}{ }^{(10)}$. The presence of the glassy phase enables them to be etched and adhesively luted to the tooth structure. CEREC Blocs (Sirona) are industrially manufactured, fine-structured feldspathic ceramic blocks used to produce inlays, onlays, crowns and veneers. They are characterized by their high polishability and enamel-like abrasion properties. Restorations also show natural appearance, as well as optimal light conducting effects and white fluorescence. CEREC Blocs integrate three different color saturation levels (chroma) and thus three different levels of translucence are present in single ceramic layer block. The enamel 
upper layer has the least pigmentation and the most translucency, the middle dentin layer has normal level of intensity, while the lower cervical layer has the strongest pigmentation and the least translucence in a similar way as natural teeth. Therefore restorations made from CEREC Blocs, they closely mimic the natural dentation without requiring any further surface characterization ${ }^{(10)}$

Lithium-disilicate CAD/CAM ceramic was introduced in the dental market in 2006 as IPS e.max CAD (Ivoclar-Vivadent). It is a monolithic type of restoration, delivered in nine shades, with a flexural strength two to three times that of the feldspathic ceramics (360 MPa to $400 \mathrm{MPa})^{(11)}$. The blocks are partially crystallized containing $0.2-1-\mu \mathrm{m}$ lithium meta-silicate crystals with approximately $40 \%$ crystals by volume. After milling, for completion of the crystallization, the restoration is fired in porcelain oven under vacuum at $850^{\circ} \mathrm{C}$ (20 to 25 minutes) ${ }^{(12)}$. Although lithium disilicate was introduced first by Ivoclar-Vivadent, yet many other companies now started to invade the market with similar products, such as the Obsidian. It is a partially crystalized lithium silicate CAD/CAM ceramic (Glidewell Laboratories), indicated for the fabrication of full-contour crowns, veneers, inlays and onlays with an average flexural strength of $385 \mathrm{MPa}$. Therefore Obsidian restorations combines both esthetics and strength with translucency that mirrors the vitality of natural teeth. ${ }^{(13)}$.

At the end of 2013, zirconia reinforced lithium silicate glass ceramic (ZLS) called "Vita Suprinity" was delivered to the market by Vita Zahnfabrik ${ }^{(14)}$. It is formed of (56-64 wt.\%) $\mathrm{SiO}_{2}$ and (8 - 12 wt. \%) $\mathrm{ZrO}_{2}$. It combines both the high mechanical properties of zirconia with flexural strength of $541 \mathrm{MPa}$ and the excellent esthetics of the lithium disilicate ${ }^{(14)}$. Vita Suprinity shows superior translucency, fluorescence and opalescence resulting in naturally appearing and biologically compatible restoration ${ }^{(15,16)}$.
Color represent one of the most important determinates that affects the success of dental restorations. The Munsell color system was the system of choice for visual measurements and color matching in dentistry. Hue, value, and chroma are the dimensions that describe color in Munsell system $^{(17)}$.

Hue, is the first dimension of color and by which we distinguish one color from another. Value, the second dimension, and it is the degree of lightness and darkness of a color in a relation to a natural gray scale extending from absolute black to absolute white ${ }^{(18)}$. While chroma, is the saturation of color $^{(19)}$.

To improve the accuracy of color reading of an object, the International Commission of I'Eclairage (CIE) has innovated many methods to formulate the spectral curves in a numerical form. The method used in dentistry is known as color space CIEL*a*b*(20). Instrumentally color space is measured and expressed in term of three coordinate value $\left(\mathrm{L}^{*}, \mathrm{a}^{*}, \mathrm{~b}^{*}\right)$ that locate the object's color within the CIELAB color space. Where $\mathrm{L}^{*}$ represent the brightness of the color or value on a numerical scale from 0 (black) to 100 (white). The color coordinate $a^{*}$ represents a position between red (when $\mathrm{a}^{*}$ has positive value) and green (when $a^{*}$ has negative value) while $b^{*}$ represent a position between yellow (when $b^{*}$ has positive value) and blue (when $b^{*}$ has negative value) ${ }^{(21,22)}$.

The color differences $(\Delta \mathrm{E})$ of two objects can then be determined by comparing the differences between respective coordinate values for each object. The formula used for calculating color differences in this system is represented as: $\Delta \mathrm{E}=$ $\left[\left(\Delta \mathrm{L}^{*}\right) 2+\left(\Delta \mathrm{a}^{*}\right) 2+\left(\Delta \mathrm{b}^{*}\right) 2\right] 1 / 2$

Where $\Delta \mathrm{L}^{*}, \Delta \mathrm{a}^{*}$ and $\Delta \mathrm{b}^{*}$ are different in color parameters for the two specimen measured for comparison ${ }^{(17)}$. 
Most of the studies have described the $\Delta \mathrm{E}$ values into two thresholds; perceptible and acceptable ${ }^{(1)}$. Kuehni et al. ${ }^{(23)}$, Seghi et al. ${ }^{(18)}$, Ruyter et al..$^{(24)}$, Acar et al. ${ }^{(25)}$ revealed that when $\Delta \mathrm{E}$ value is $1,50 \%$ of observers accepted the color and when $\Delta \mathrm{E}$ value is below 1, the eye will not perceive the color change. However, if the $\Delta \mathrm{E}$ is above 1 , it will be perceived by human eye. While Johnston and $\mathrm{Kao}^{(26)}$ claimed that if $\Delta \mathrm{E}$ is below 3.7 , the restoration will be clinically acceptable and if the $\Delta \mathrm{E}$ is above this value, this will lead to clinically unacceptable and unaesthetic restoration.

The oral cavity is a changeable environment, due $\mathrm{pH}$ fluctuation. The consumption of beverages frequently, such as coffee, tea and soft drinks may affect the color of dental restorative materials due to the difference in their chemical composition. They may discolor or disintegrate due to the absorption of substances from saliva ${ }^{(27)}$. Therefore color matching and long-lasting color stability of dental materials are two of the major factors that influence the success or the failure of any esthetic dental restoration. Thus, the purpose of this study was to evaluate the color stability of four different $\mathrm{CAD} / \mathrm{CAM}$ ceramics after immersion in coffee solution and the null hypothesis was that there will be no difference in color stability between the four different ceramic materials after their immersion in coffee solution.

\section{MATERIALS AND METHODS}

The materials used in the present study are presented in Table 1:

TABLE (1) Commercial names, types, chemical compositions, and manufacturer of different materials used in this study:

\begin{tabular}{|c|c|c|c|c|}
\hline $\begin{array}{c}\text { Materials } \\
\text { (Commercial names) }\end{array}$ & $\begin{array}{c}\text { Type/ } \\
\text { Description }\end{array}$ & Chemical composition & (In $\%$ by weight) & Manufacturer \\
\hline IPS e.max CAD & $\begin{array}{l}\text { Lithum } \\
\text { disilicate glass- } \\
\text { ceramic block }\end{array}$ & $\begin{array}{l}\mathrm{SiO}_{2} \\
\mathrm{Li}_{2} \mathrm{O} \\
\mathrm{K}_{2} \mathrm{O} \\
\mathrm{P}_{2} \mathrm{O}_{2} \\
\mathrm{ZrO}_{2} \\
\mathrm{ZnO} \\
\text { Other } \\
\text { Colouring oxides }\end{array}$ & $\begin{array}{l}57.0-80.0 \\
11.0-19.0 \\
0.0-13.0 \\
0.0-11.0 \\
0.0-8.0 \\
0.0-8.0 \\
0.0-10.0 \\
0.0-8.0\end{array}$ & $\begin{array}{l}\text { Ivoclar Vivadent, } \\
\text { Schaan, } \\
\text { Liechtenstein. } \\
\text { canada }\end{array}$ \\
\hline Vita Suprinity & $\begin{array}{c}\text { Zirconia } \\
\text { reinforced } \\
\text { lithium silicate }\end{array}$ & $\begin{array}{l}\mathrm{ZrO}_{2} \\
\mathrm{SiO}_{2} \\
\mathrm{Li}_{2} \mathrm{O} \\
\text { Various }>10\end{array}$ & $\begin{array}{l}8-12 \\
56-64 \\
15-21\end{array}$ & $\begin{array}{c}\text { VITA Zahnfabrik, } \\
\text { Germany. }\end{array}$ \\
\hline Cerec Blocs & $\begin{array}{c}\text { Feldspathic } \\
\text { ceramic }\end{array}$ & $\begin{array}{l}\mathrm{SiO}_{2} \\
\mathrm{Al}_{2} \mathrm{O}_{3} \\
\mathrm{Na}_{2} \mathrm{O} \\
\mathrm{K}_{2} \mathrm{O} \\
\mathrm{CaO} \\
\mathrm{TiO}_{2}\end{array}$ & $\begin{array}{l}56-64 \\
20-23 \\
6-9 \\
6-8 \\
0,3-0,6 \\
0,0\end{array}$ & Sirona, USA \\
\hline
\end{tabular}




\begin{tabular}{|c|c|c|c|}
\hline Obsidian & Lithium silicate & $\begin{array}{ll}\mathrm{SiO}_{2} & 52-59 \\
\mathrm{Li}_{2} \mathrm{O} & 15-20 \\
\mathrm{ZrO}_{2} & 2-10 \\
\mathrm{Al}_{2} \mathrm{O}_{3} & 2-5 \\
\mathrm{~K}_{2} \mathrm{O} & 2-5 \\
\mathrm{Na}_{2} \mathrm{O} & 2-3 \\
\mathrm{GeO}_{2} & 0-2 \\
\text { Others and coloring oxides } & 0-12\end{array}$ & $\begin{array}{c}\text { Glidewell } \\
\text { Laboratories }\end{array}$ \\
\hline $\begin{array}{c}\text { Filtek }^{\mathrm{TM}} \mathrm{Z} 250 \\
\text { Universal Restorative } \\
\text { Dental Composite. }\end{array}$ & $\begin{array}{l}\text { composite resin, } \\
\text { available in } 4 \mathrm{~g} \\
\text { syringe }\end{array}$ & $\begin{array}{l}\text {--Silane Treated Ceramic } 75 \text { - } 85 \\
\text {-Bisphenol A Polyethylene Glycol Diether, Dimethacrylate } \\
5 \text { - 10, } \\
\text {-Diurethane Dimethacrylate, } \\
\text {-Bisphenol A Diglycidyl Ether Dimethacrylate (Bis-GMA), } \\
\text {-Triethylene Glycol Dimethacrylate (TEGDMA) <5, } \\
\text {-Water. } \\
\text { Base: Methacrylate monomers containing phosphoric acid } \\
\text { groups, Methacrylate monomers, Silanated fillers, Initiator } \\
\text { components, Stabilizers, Rheological additives. } \\
\text { Catalyst: Methacrylate monomers, Alkaline (basic) fillers, } \\
\text { Silanated fillers, Initiator components, Stabilizers, Pigments, } \\
\text { Rheological additives. }\end{array}$ & $\begin{array}{c}\text { 3M ESPE }{ }^{\mathrm{TM}}, \\
\text { Seefeld, Germany }\end{array}$ \\
\hline $\begin{array}{l}\text { RelyX }{ }^{\mathrm{TM}} \mathrm{U} 200 \\
\text { Automix. }\end{array}$ & $\begin{array}{c}\text { Dual-Cure } \\
\text { Self- Adhesive } \\
\text { Resin Cement. }\end{array}$ & $\begin{array}{l}\text { Base: Methacrylate monomers containing phosphoric acid } \\
\text { groups, Methacrylate monomers, Silanated fillers, Initiator } \\
\text { components, Stabilizers, Rheological additives. } \\
\text { Catalyst: Methacrylate monomers, Alkaline (basic) fillers, } \\
\text { Silanated fillers, Initiator components, Stabilizers, Pigments, } \\
\text { Rheological additives. }\end{array}$ & 3M ESPE, Germany \\
\hline
\end{tabular}

A total of twenty ceramic disc shaped samples with shade A2 $(10 \mathrm{~mm}$ in diameter and $2 \mathrm{~mm}$ in thickness) were designed and constructed in standardized manner. Samples were divided into four main equal groups $(n=5)$ each, according to the type of ceramic material used. Group I: IPS e.max CAD. Group V: Vita Suprinity. Group C: Cerec Blocs. Group O: Obsidian

All specimens were fabricated using $\mathrm{CNC}$ milling machine and electric isoMet microsaw 4000. They were seated in central motor machine of the Computer numerically controlled (CNC Jr.
Table Top Mill XD Series Vertical CNC Milling Machine, CNC Masters, USA) Milling/machining technology which fabricates restorations utilizing subtraction-manufacturing technology from large solid blocks using a sharp abrasive stone to mechanically cut the material in slices. The motor moved in two directions; rotation on $\mathrm{X}$ axes and forward motion on $\mathrm{Y}$ axes. This forward movement is directed to make the abrasive stone mill the block. All specimens were then cut into discs from cylinder ceramic blocks using the electric isoMet 4000 microsaw (Buehler, USA). Cutting the samples in $2 \mathrm{~mm}$ thickness was done using saw diamond 
disk (Renfert GmbH, Germany) 0.7mm thickness Buehler at a speed of $2500 \mathrm{rpm}$ under water coolant, and feeding rate of $5 \mathrm{~mm} / \mathrm{min}$ to reach disks of $(10 \times 2 \mathrm{~mm})$ in dimension (figure 1). A digital caliper was used to verify the dimension after each cutting process.

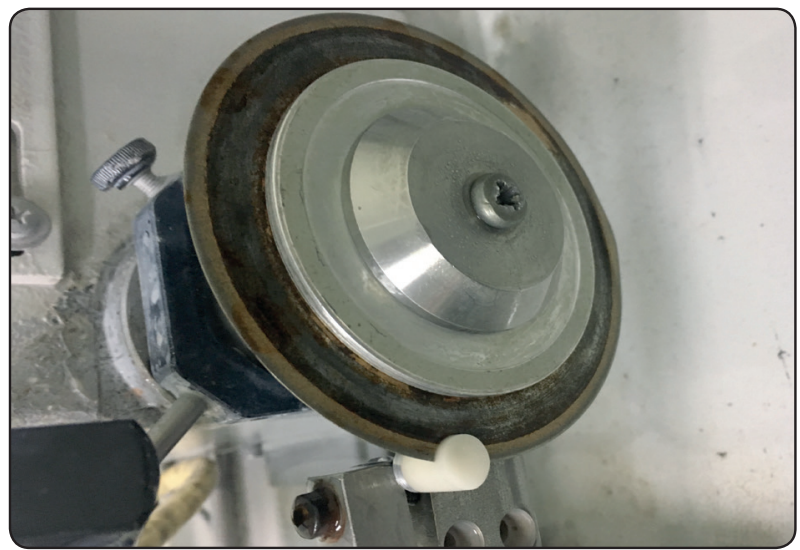

Fig. (1) Cutting ceramic block into disc shaped samples using electric isoMet 4000 microsaw.

\section{IPS e.max CAD, Vita Suprinity and Obsidian crystallization:}

Five IPS e.max CAD disc samples were placed in the firing furnace (Programat P500 furnace; Ivoclar-Vivadent, Schaan, Liechtenstein, Canada). The samples were pre-dried under vacuum at $403^{\circ}$ $\mathrm{C}$ for 6 minutes then the heating temperature was increased at a rate of $90^{\circ} \mathrm{C} / \mathrm{min}$ until a temperature of $820^{\circ} \mathrm{C}$ was reached and held for 10 minutes. The temperature was further increased at a rate of $30^{\circ} \mathrm{C} /$ min until a firing temperature of $850^{\circ} \mathrm{C}$ was obtained and held for 7 minutes to complete the crystallization of the lithium disilicate. Vita Suprinity disc samples were also placed in Programat P500 furnace and pre-dried at $400^{\circ} \mathrm{C}$ for 4 minutes then the heating temperature was increased at a rate of $90^{\circ} \mathrm{C} / \mathrm{min}$ until a temperature of $820^{\circ} \mathrm{C}$ was reached and held for 10 minutes. Then the temperature was increased at a rate of $30^{\circ} \mathrm{C} / \mathrm{min}$ until a firing temperature of $850^{\circ} \mathrm{C}$ was obtained and held for 7 minutes to complete the crystallization. After crystallization of both materials has been completed the furnace was opened and the samples were left inside the furnace on the try to cool down for 10-15 minutes.

The Obsidian disc samples were pre-dried under vacuum at $400^{\circ} \mathrm{C}$ for 3 minutes, then the heating temperature was increased at a rate of $90^{\circ} \mathrm{C} / \mathrm{min}$ until a temperature of $780^{\circ} \mathrm{C}$ was reached and held for 10 seconds. A second heating rate was performed $40^{\circ} \mathrm{C} / \mathrm{min}$ until a temperature of $820^{\circ} \mathrm{C}$ was reached and held for 10 minutes to complete the crystallization. Then long term cooling at a rate of $50^{\circ} \mathrm{C} / \mathrm{min}$ was performed.

\section{Substrate construction}

A specially constructed cylindrical split teflon mold was fabricated. The mold has a circular central hole $10 \mathrm{~mm}$ in diameter and $2 \mathrm{~mm}$ in thicknesses, with an outer cupper ring that served for the assembling of the two halves of the teflon mold. Twenty composite resin discs A3 shade were fabricated according to manufacturer's instructions. A thin layer of separating medium was applied on the teflon mold that was seated on a dry clean glass slab. Composite resin was applied incrementally, each increment was $1 \mathrm{~mm}$ in thickness using a nonmetallic plastic instrument. After application of the second increment, a mylar strip was pressed on the glass plate in order to provide optimum smoothness. A LED light curing unit (Miraj, LED.D curing light, Korea) with a mean light intensity of $1400 \mathrm{~mW} / \mathrm{cm} 2$ and optical wavelength of 420-480 nm was used for composite resin activation for 40 seconds.

\section{Cementation procedure}

The cementing surface of the ceramic disc samples were etched with Ultradent porcelain etching gel (9\% hydrofluoric acid) for 20 seconds according to manufacturer's recommendations. The etched surfaces were thoroughly rinsed using water spray for 60 seconds, followed by ultrasonic cleansing in distilled water for another 60 seconds, then dried by oil-free compressed air for 
30 seconds. A layer of silane coupling agent (Ultradent Products, Inc.) was applied to the etched surface for 60 seconds followed by air thinning.

The cemented side of composite resin discs were manually finished using wet silicon carbide paper 320,600 grit (Norton S.A., São Paulo, Brazil) then washed with tap water for 1 minute, and ultrasonically cleaned in distilled water for 10 minutes.

A specially designed cementation device was machined from stainless steel in order to ensure standard load application, it consists of 1 . Cementation mold that is used to hold the samples together inside the base of the cementation device, the mold is formed of 4 parts: a) Teflon part: Two teflon halves with circular central hole $10 \mathrm{~mm}$ inner diameter and $2 \mathrm{~mm}$ thickness, to hold the composite substrate. b) Metal part: circular in shape with square central hole to allow for escapement of excess cement, $10 \mathrm{~mm}$ inner side, and $0.5 \mathrm{~mm}$ thickness, to create space for the cement. c) Metal part: circular in shape with central hole $10 \mathrm{~mm}$ inner diameter and $2 \mathrm{~mm}$ thickness, to hold the ceramic discs in centralized position in relation to a composite substrate. d) Teflon ring: with $35 \mathrm{~mm}$ outer diameter and $27 \mathrm{~mm}$ inner circumference diameter, to aid in assembling the three parts ( $a, b$ and $c)$ together during loading (figure 2,3). 2. Cementation device (figure 4) that consists of 4 parts: a) Two horizontal metal plates rectangular in shape (upper and lower) with sample fixation screws connected together with two vertical metal arms attached to both plates. b) Two supporting vertical metal arms attached to the upper and lower horizontal metal plates. c) A T shaped metallic rod is attached to the upper metal plates and can move freely vertically, it also carries at its upper end a disc shaped plate over which the required load will be placed, while at its lower end a teflon rod with $10 \mathrm{~mm}$ diameter tip was attached. d) A $2 \mathrm{Kg}$ load was placed on the disc shaped plate of part (c).

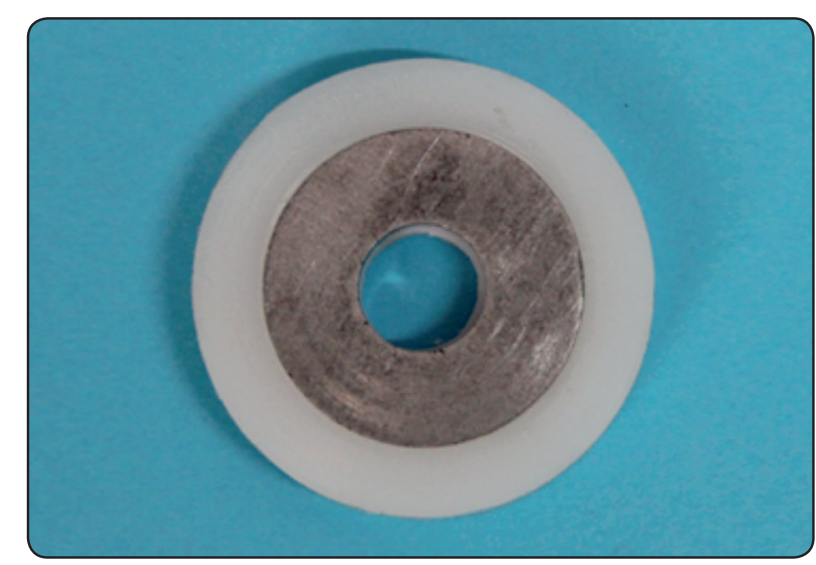

Fig. (2) Assembled cementation mold.

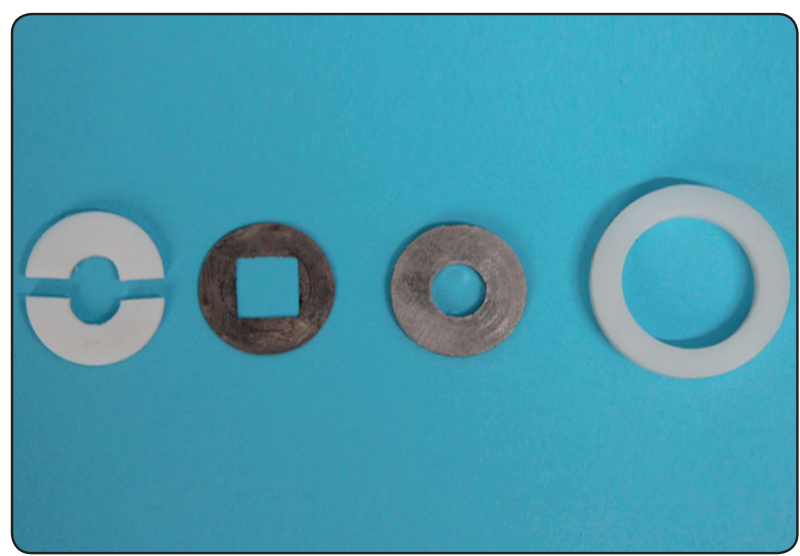

Fig. (3) Dissassembled Cementation mold with outer teflon ring.

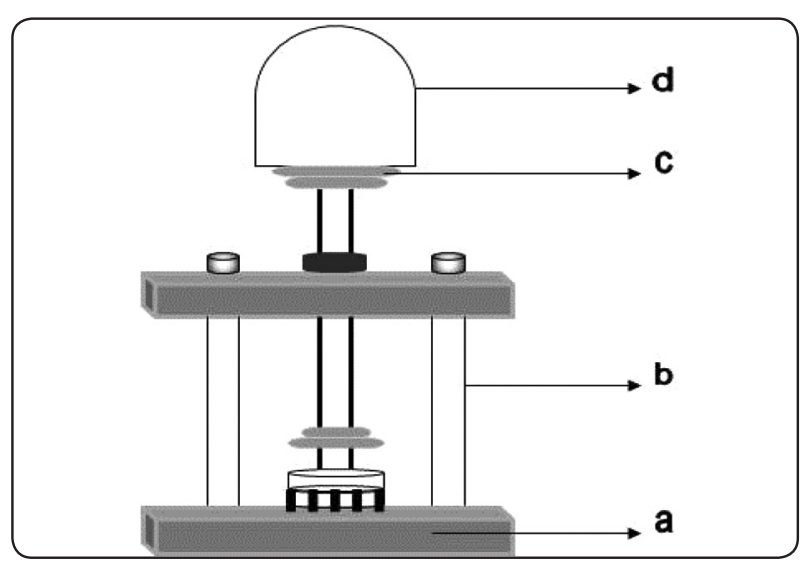

Fig. (4) Schematic diagram for the cementation device. 
Cementation was accomplished according to the manufacturer instructions. Dual cure Rely X U200 Automix self-adhesive resin luting agent shade A2 was used. The cement was injected from the doublepush syringe and automixed within the mixing tips, to the prepared surface of each composite substrate.

The cementation mold was placed on the rectangular shaped metallic base of the cementation device, and then secured in centralized position by fixation screw to ensure its placement in the same location each time during cementation. Each composite disc was positioned inside the teflon mold cavity part (a) with its prepared surface facing the luting cement. The metal part (b) was then placed over part (a), and then part (c) was placed over them to secure the ceramic disc in place. Finally, teflon ring accommodated the three parts $(\mathrm{a}, \mathrm{b}$ and c) together during loading.

A $2 \mathrm{Kg}$ constant load was applied on the disk shaped plate at the upper end of the T-shaped metallic part of the cementation device, and was left for 3 minutes. The excess cement was then removed with a sharp probe from the corner of the square hole of metal part (b), the resin material was polymerized from three directions for 40 seconds with light cure device at a power of $3.200 \mathrm{~mW} / \mathrm{cm} 2$.

After completion of curing, the outer teflon ring was removed to disassemble the cementation mold parts, and the cemented ceramic discs were removed, then finishing was made using finishing bur.

Finishing and polishing of all ceramic disc samples were made according to manufacturer recommendations.

For standardization, finishing and polishing were done with the same technician, till a flat surface was achieved which is necessary for color parameters measurement to allow the contact tip of the spectrophotometer to contact the surface without any angulation.
All samples were immersed in saline solution for 24 hours, prior to baseline assessment. The samples were then removed using a tweezer and blotted dry using filter paper and immersed in a coffee solution.

A 5 g Nescafé coffee powder ( $\mathrm{PH}=5.8$, Nestlé, Switzerland) was dissolved in $200 \mathrm{~mL}$ boiling distilled water, stirred for 10 minutes then filtered through a filter paper.

Samples were kept at a constant temperature of $50^{\circ} \pm 1^{\circ} \mathrm{c}$ in an incubator (Incubator: Shanghai Boxun Industry \& commerce Co., Ltd. BPX Series. Edition 03-2012. Medical Equipment Factory) for 3 weeks, then were removed by a tweezer, washed under runny distilled water, and dried.

All disc samples were tested twice; before (baseline) and after immersion in coffee solution. The color of each sample was assessed using a spectrophotometer (Spectrophotometer: Agilent Cary 5000 UV-Vis-NIR Spectrophotometer, USA, National Institute for Standards, Cairo).

CIE-Lab color values for each sample were calculated from the diffuse reflectance data by using the color software application which is available through Cary WinUv instrument and supports the extensive color calculations and standards. The $\mathrm{L}^{*}$ $a^{*} b^{*}$ values of the samples were recorded according to the CIELAB color scale relative to the standard illumination D65.

Where $\mathrm{L}^{*}$ is a measure of the Lightness of an object, ranging from 0 (Black) to 100 (White), $a^{*}$ is a measure of redness $(a>0)$ or greenness $(a<0), b^{*}$ is a measure of yellowness $(b>0)$ or blueness $(b<0)$. Along the vertical axis (the neutral axis), between black $(\mathrm{L}=0)$ and white $(\mathrm{L}=100)$ is a continuous range of gray shades.

\section{Color difference determination}

The degree of color difference between the compared colors is expressed in $\Delta \mathrm{E}$ units. The total color difference, according to $\mathrm{L}^{*}, \mathrm{a}^{*}, \mathrm{~b}^{*}$ 
coordinates, is calculated as shown in the equation $\Delta \mathrm{E}^{*} \mathrm{ab}=\left((\Delta \mathrm{L} *) 2+\left(\Delta \mathrm{a}^{*}\right) 2+\left(\Delta \mathrm{b}^{*}\right) 2\right) 1 / 2$, where $\Delta \mathrm{E}$ is the color difference between baseline color and the color of the immersed disc samples, $\Delta \mathrm{L}^{*}$ refer to the difference in lightness, $\Delta \mathrm{a}^{*}$ and $\Delta \mathrm{b}^{*}$ refer to the difference in chromaticity values between the baseline and immersed readings of the discs.

\section{Statistical analysis}

Numerical data were explored for normality by checking the data distribution and using Kolmogorov-Smirnov and Shapiro-Wilk tests. All data showed parametric distribution except for $(\Delta L)$ values. Data were represented as mean, standard deviation (SD), median, range and 95\% Confidence interval $(95 \% \mathrm{CI})$ values.

For parametric data, one-way ANOVA followed by Tukey's post-hoc test was used to compare between the four groups. For non-parametric data, Kruskal-Wallis test was used to compare between the four groups.

The significance level was set at $\mathrm{P} \leq 0.05$. Statistical analysis was performed with IBM ${ }^{\circledR}$ SPSS ${ }^{\circledR}$ Statistics Version 20 for Windows.

\section{RESULTS}

Descriptive statistics and results of comparison between changes in different color parameters are presented in Table (2) and figure (5).

As regards $(\Delta \mathrm{L})$, there was a statistically significant difference between the four ceramic groups after immersion in coffee solution. All groups showed a decrease in $\left(\mathrm{L}^{*}\right)$ values. Pair-wise

TABLE (2): Descriptive statistics and results of one-way ANOVA and Kruskal-Wallis tests for comparisons between changes in color parameters among the four different ceramic groups.

\begin{tabular}{|c|c|c|c|c|c|c|c|c|c|}
\hline \multirow{2}{*}{ Parameter } & \multirow{2}{*}{ Ceramic type } & \multirow{2}{*}{ Mean } & \multirow{2}{*}{$\mathrm{SD}$} & \multirow{2}{*}{ Median } & \multirow{2}{*}{ Minimum } & \multirow{2}{*}{ Maximum } & \multicolumn{2}{|c|}{$95 \% \mathrm{CI}$} & \multirow{2}{*}{$P$-value } \\
\hline & & & & & & & Lower bound & Upper bound & \\
\hline \multirow{4}{*}{$\Delta \mathrm{L}$} & Cerec & $-2.06^{b}$ & 1.17 & -1.82 & -4.05 & -0.97 & -3.51 & -0.61 & \multirow{4}{*}{$0.006 *$} \\
\hline & Obsidian & $-1.48^{b}$ & 1.21 & -1.59 & -3.29 & 0.00 & -2.99 & 0.03 & \\
\hline & Suprinity & $-2.04^{b}$ & 0.31 & -1.94 & -2.43 & -1.74 & -2.43 & -1.66 & \\
\hline & e.max CAD & $-5.19^{a}$ & 0.71 & -5.09 & -6.05 & -4.17 & -6.08 & -4.31 & \\
\hline \multirow{4}{*}{$\Delta \mathrm{a}$} & Cerec & 3.07 & 0.12 & 3.08 & 2.88 & 3.18 & 2.92 & 3.22 & \multirow{4}{*}{0.695} \\
\hline & Obsidian & 3.18 & 0.13 & 3.22 & 3.03 & 3.30 & 3.01 & 3.35 & \\
\hline & Suprinity & 2.93 & 0.18 & 2.94 & 2.69 & 3.18 & 2.70 & 3.15 & \\
\hline & e.max CAD & 3.07 & 0.61 & 3.24 & 2.01 & 3.49 & 2.31 & 3.83 & \\
\hline \multirow{4}{*}{$\Delta \mathrm{b}$} & Cerec & $4.34^{\mathrm{b}}$ & 0.18 & 4.23 & 4.21 & 4.63 & 4.11 & 4.56 & \multirow{4}{*}{$<0.001 *$} \\
\hline & Obsidian & $5.30^{\mathrm{a}}$ & 0.15 & 5.22 & 5.17 & 5.52 & 5.12 & 5.49 & \\
\hline & Suprinity & $4.77 \mathrm{ab}$ & 0.52 & 4.84 & 3.95 & 5.34 & 4.12 & 5.42 & \\
\hline & e.max CAD & $2.04^{\mathrm{c}}$ & 0.49 & 1.91 & 1.57 & 2.86 & 1.43 & 2.64 & \\
\hline \multirow{4}{*}{$\Delta \mathrm{E}$} & Cerec & 5.78 & 0.49 & 5.57 & 5.47 & 6.65 & 5.17 & 6.39 & \multirow{4}{*}{0.250} \\
\hline & Obsidian & 6.44 & 0.40 & 6.31 & 6.10 & 7.13 & 5.95 & 6.94 & \\
\hline & Suprinity & 5.97 & 0.45 & 5.90 & 5.31 & 6.52 & 5.41 & 6.53 & \\
\hline & e.max CAD & 6.39 & 0.88 & 6.62 & 4.89 & 7.11 & 5.30 & 7.48 & \\
\hline
\end{tabular}

*: Significant at $P \leq 0.05$, Different superscripts are statistically significantly different 


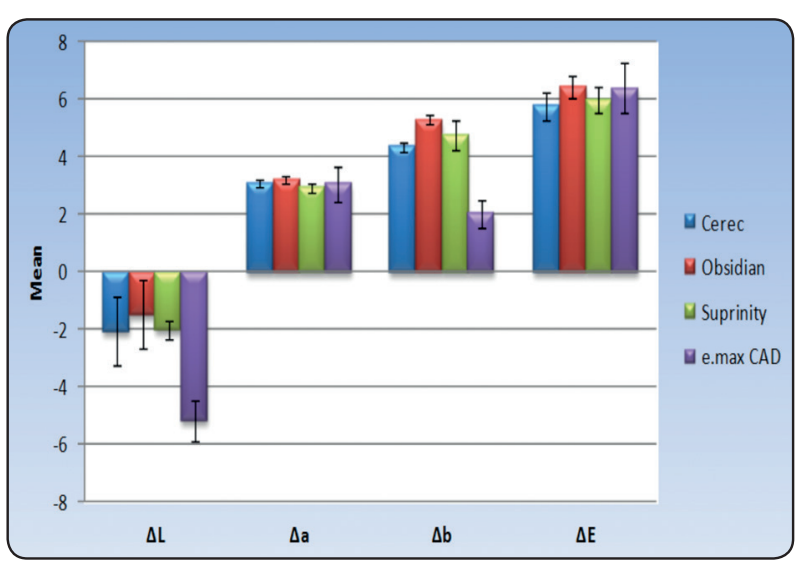

Fig. (5) Bar chart representing mean and standard deviation values of color changes among the four different ceramic groups.

comparisons revealed that IPS e.max CAD showed the highest mean decrease in $(\Delta \mathrm{L})(-5.19 \pm 0.71)$. While there was no statistically significant difference between the mean $(\Delta \mathrm{L})$ of Cerec Blocs, Obsidian and Vita Suprinity respectively $(-2.06 \pm 1.17$; $-1.48 \pm 1.21 ;-2.04 \pm 0.31)$ all showed lower decrease in $\left(\mathrm{L}^{*}\right)$ value than IPS e.max CAD.

For $(\Delta b)$, there was a statistically significant difference between the four ceramic groups after immersion in coffee solution. Pair-wise comparisons revealed that Obsidian showed the highest mean ( $\Delta$ b) $(5.30 \pm 0.15)$ with non-statistically significant difference from Vita Suprinity $(4.77 \pm 0.52)$. Cerec Blocs showed lower mean $(\Delta \mathrm{b})(4.34 \mathrm{~b} \pm 0.18)$ with non-statistically significant difference from Vita Suprinity but with a statistically significant difference from Obsidian. IPS e.max CAD showed the statistically significantly lowest mean $(\Delta b)$ (2.04 \pm 0.49$)$.

For $(\Delta \mathrm{a})$ and $(\Delta \mathrm{E})$ no statistically significant difference was found among the four different ceramic groups (Cerec Blocs, Obsidian, Vita Suprinity, IPS e.max CAD) respectively after immersion in coffee solution where mean $(\Delta a)$ was $(3.07 \pm 0.12 ; 3.18 \pm 0.13 ; 2.93 \pm 0.18 ; 3.07 \pm 0.61)$ and mean $(\Delta \mathrm{E})$ was $(5.78 \pm 0.49 ; 6.44 \pm 0.40 ; 5.97 \pm 0.45$; $6.39 \pm 0.88)$.

\section{DISCUSSION}

The continuous increase in esthetic awareness has led to rapid development in the art and science of ceramic dental materials where patients demand for restorations of natural appearance became mandatory ${ }^{(4,28)}$. Chairside CAD/CAM systems are considered nowadays an accurate and efficient technology that meet the patient's esthetic requirements ${ }^{(29,5)}$.

In the present study, four types of CAD/CAM ceramic materials were selected due to their reported advantages as claimed by the manufacturer. IPS e.max CAD (lithium disilicate) was selected due to its excellent machinability, polishability and esthetics, together with high flexural strength of 360 to $400 \mathrm{MPa}{ }^{(30)}$. Vita Suprinity (zirconia reinforced lithium silicate) with 8-12 Wt. \% zirconia was used in this study due to its excellent esthetics, translucency, opalescence, fluorescence and high flexural strength (540 MPa) ${ }^{(31)}$. Obsidian (lithium silicate) ceramic was also selected due to its natural vitality, toughness and flexural Strength of $385 \mathrm{MPa}$ that surpasses conventional ceramics ${ }^{(13)}$. Cerec Blocs was favored in this study as being a category of $\mathrm{CAD} / \mathrm{CAM}$ feldspathic porcelain known to reproduce the color gradients characteristic of natural teeth ${ }^{(10)}$.

Discoloration of dental ceramics in the oral cavity due to the continuous use of strong coloring beverages as coffee are crucial and it may affect the esthetics longevity of any restoration. Therefore this study aimed to evaluate the color stability of the four previously mentioned CAD/CAM ceramics after immersion in coffee solution.

In the present investigation, ceramic blocks were fabricated under controlled and optimum manufacturer conditions ${ }^{(32,11)}$. Moreover, CNC milling machine and electric isoMet microsaw 4000 systems were selected to construct the disc samples, as they offer the opportunity to prepare the ceramic blocks to their desired shape and they are considered a reliable method in research work according to the methodology followed by Arocha et al. ${ }^{(33)}$ in 2014. 
In this study, $2 \mathrm{~mm}$ ceramic discs thickness were determined, which was reported to be the maximum recommended thickness for fixed restoration. While $10 \mathrm{~mm}$ diameter was selected, corresponding to the spectrophotometer sample compartment size that allows the testing machine to record the readings easily and accurately ${ }^{(33,34)}$. The composite substrate was also made at the same dimensions to provide symmetry between the two discs. In order to closely mimic the clinical situation the ceramic discs were luted to the composite resin substrate (shade A3) resembling the dentine structure ${ }^{(35)}$. For standardization a specially designed cylindrical split teflon mold was made for the construction of composite substrate with circular central hole 10 $\mathrm{mm}$ in diameter and $2 \mathrm{~mm}$ in thickness ${ }^{(35)}$.

The cemented side of all discs was acid etched to increase the surface area for bonding and provides micro-mechanical retention. Silane coupling agent was then applied, being a biofunctional molecule that bonds to both the ceramic silica and the organic part of the resin, thus enhancing the bond strength by increasing the wettability ${ }^{(36)}$.

The selection of the luting agent is a critical factor for obtaining optimal esthetics with ceramic restorations. Therefore in this study, self-adhesive (RelyX $^{\mathrm{TM}}$ U200 Automix) cement was chosen due to its excellent color stability, moisture tolerance, high bond strength to all substrates and superior mechanical properties ${ }^{(37)}$. Being automix ensure that the cement was free of voids and has a standardized mix. Shade A2 was selected to be similar to that of the shade of the ceramic discs. Also a specially formulated cementing device was used to standardize the thickness for all specimens.

In the present study, a special cementation device was fabricated to lute the ceramic discs to their corresponding composite discs. Cementation was done under a constant $2 \mathrm{Kg}$ load for standardization $^{(35)}$.

Finishing and polishing of the ceramic samples was done following the manufacturer recommendations of each company and was performed by the same technician for standardization purpose till achieving flat surfaces ${ }^{(38)}$.

The cemented samples were immersed in coffee solution for three weeks which was stated to be equivalent to drinking 1 cup of coffee per day for 1 year ${ }^{(39)}$. For the color assessment, spectrophotometer was used as it eliminates the subjective interpretation of visual color comparison and can detect any slight color changes in dental materials. Therefore it is considered an accurate instrument for color measurement in dentistry ${ }^{(39,40)}$.

Summarizing the results of the present study requires total rejection of the null hypothesis where all types of ceramic materials showed color instability after immersion in coffee solution.

The results of this study revealed non-statistically significant difference among the four different ceramic materials (IPS e.max CAD, Cerec Blocs, Obsidian, Vita Suprinity) after immersion in coffee solution, and $\Delta \mathrm{E}$ of all types of ceramics were above 3.7 which is clinically perceivable by human eye and thus is considered to be clinically unacceptable and unaesthetic. The mean $\triangle \mathrm{E}$ of IPS e.max CAD was 6.39, of Cerec Blocs was 5.78, of Obsidian was 6.44 and of Vita Suprinity was 5.97. These findings could be attributed to the fact that coffee contains yellow colorants, that might be eluted later ${ }^{(39,41)}$ and resulted in extrinsic discoloration. These findings were in agreement with Alharbi et al. ${ }^{(42)}$, Awad et al. ${ }^{(43)}$, Ataya et al. ${ }^{(44)}$, and Acar et al. ${ }^{(25)}$, who proved that the greatest color changes were seen in restorations when immersed in coffee solution.

The results were also in accordance with Ghahramanloo et al. ${ }^{(45)}$ who showed that common beverages like red wine, tea, and coffee induce significant color changes in composite resins and dental porcelains. Nevertheless, they revealed that these beverages only induce external staining and thus their effects on the internal structure must be further evaluated. 
However, the results of the present study were not in accordance with Gawriołek et al. ${ }^{(46)}$ who found that color changes due to staining staining of lithium disilicate material was not visually perceivable and that ceramic materials exhibited better color stability than composite resins. With Samra et al. ${ }^{(41)}$ who stated that $\Delta \mathrm{E}$ values of empress 2 was 1.28 and that it displayed the lowest discoloration when compared to composite materials after 15 days immersion in coffee.

The different results of the present investigation to previous ones could be related to the methodology applied in this study. Based on the fact that the ceramics used being all translucent reflecting the color of the underneath and that Filtek ${ }^{\mathrm{TM}}$ Z250 composite resin was used as a substrate material to resemble the dentine structure aiming to mimic as close as possible the clinical situation. Composite material however was found to undergo both extrinsic and intrinsic discoloration when immersed in coffee solution through adsorption of colorants on the surface and the sub-surface. These findings were related to compatibility of the polymeric resin matrix with yellow coffee colorants ${ }^{(47,48)}$ which therefore explains the results of the present study where an increase in values of the $b^{*}$ coordinate tending to be yellow was found. The results might be also related to the chemical nature of composite resin material used in the study being formed of hydrophilic triethylene glycol dimethacrylate (TEGDMA). TEGDMA undergoes higher water absorption and thus allowing hydrophilic colorant to penetrate into the resin matrix. Additionally, the presence of bisphenol A-glycidyl methacrylate (BisGMA) which was found to cause the highest water sorption compared with UDMA, TEGDMA ${ }^{(49-51)}$. These findings were in agreement with Bagheri et al. ${ }^{(52)}$ who reported that resin composites containing TEGDMA in their resin matrix discolor more than other types of composites containing UDMA. With Kugel ${ }^{(53)}$ and Moszner et al. ${ }^{(54)}$ who stated that resin matrix of dental composite materials plays a major role in stain susceptibility and that UDMA are more stain-resistant than Bis-GMA. The results of the present study revealed a decrease in $\Delta \mathrm{L}$ and the four types of ceramics became darker. These findings could be attributed to the material itself. The ceramic materials selected had high translucency due to the optical combination of a glass matrix and crystalline phase that reduces the internal scattering of light as it passes through them. As a result, the underlying color of the resin structure might have darken the final color of the resulting restoration ${ }^{(55)}$.

The results of this study showed a statistically significant difference between the four ceramic groups after immersion in coffee solution in $\Delta b$ where IPS e.max CAD and Cerec Blocs showed lower mean values than both Obsidian and Vita Suprinity ceramics. These findings were in accordance with Samara et al ${ }^{(56)}$ who stated that difference in chemical composition and surface texture of dental porcelain has an effect on the amount of adsorption of yellow pigments and thus resulting in different $b^{*}$ parameter. The result of this study could be also related to surface finish of ceramic restorations where polishing was made instead of glazing. Although polishing has been previously investigated and even proved to be an efficient alternative to surface glazing to produce a smooth surface to dental ceramics it's still a matter of controversy and a subject for research to differentiate between polishing and glazing of dental ceramics and their effect on color stability and final strength ${ }^{(38)}$.

One of the limitations of this in vitro study was that ceramic materials were exposed to staining solution on both sides, while clinically any dental restoration is bonded to tooth structure and is subjected to oral beverages and coloring agents only in one side. The specimens were also bonded to composite substrate rather tooth structure and this might have affected the final color due the staining capabilities of resin materials. Further clinical studies are therefore needed to evaluate the susceptibility of dental ceramics to staining by different types of oral beverages and coloring agents. 


\section{CONCLUSIONS}

Within the limitation of this study it can be concluded that the color stability of the four tested CAD/CAM ceramics was affected by immersion in coffee solution and they became darker in appearance.

\section{REFERENCES}

1. Zarone F, Russo S, Sorrentino R. From porcelain-fused-tometal to zirconia: Clinical and experimental considerations. Dental materials. 2011; 27:83-96.

2. Christensen GJ. Porcelain-fused-to-metal versus zirconiabased ceramic restorations. J Am Dent Assoc 2009; 140(8):1036-9

3. Conrad H, Seong WJ, Pesun IJ. Current ceramic materials and systems with clinical recommendations: A systematic review. J Prosthet Dent 2007; 98:389-404.

4. Denry I and Holloway JA. Ceramics for dental applications: a review. Materials 2010;3: 351-368.

5. Miyazaki T1, Hotta Y, Kunii J, Kuriyama S, Tamaki Y. A review of dental CAD/CAM: current status and future perspectives from 20 years of experience. . Dent Mater J. 2009 Jan; 28(1):44-56.

6. Kelly JR, Nishimura I, Campbell SD. Ceramics in dentistry: historical roots and current perspectives. J Prosthet Dent. 1996; 75(1): 18-32.

7. Alghzzawi TF. Advancements in CAD/CAM technology: Options for practical implementation J Prosthodont Res. 2016 Apr; 60(2):72-848

8. Hamza TA and sherif RM. In-vitro evaluation of the marginal fit of monolithic zirconia restorations fabricated with different CAD/CAM systems. J prosthet Dent 2016 in press

9. Sato K, Matsumura H, Atsuta M. relation between cavity design and marginal adaptation in machine-milled ceramic restorative system. J Oral Rehabil 2002;29:24-7.

10. Han GJ, Kim JH, Lee MA, Chae SY, Lee YH, Cho BH. Performance of a novel polishing rubber wheel in improving surface roughness of feldspathic porcelain. Dent Mater J. 2014; 33(6):739-48. Epub 2014 Oct 11.

11. Charlton DG, Robert HW, Tiba A. Measurement of select physical and mechanical properties of 3 machinable ceramic materials. Quintessence Int. 2008; 39(7):573-579.
12. IPS e.max lithium disilicate: the future of all-ceramic dentistry. Material science, practical applications, keys to success. Ivoclar publication 627329. March 2009:1-15.

13. Performance Evaluation of CEREC® MC XL Milling System Utilizing Obsidian ${ }^{\circledR}$ Milling Blocks. February 25 , 2016.

14. Morsi TS and Ghanem L. Effect of artificial accelerated aging on flexural strength, microstructure and colour of a Zirconia reinforced Lithium silicate ceramic. AinShams D.J 2016; 19:22-34.

15. Elsaka SE and Elnaghy AM. Mechanical properties of zirconia reinforced lithium silicate glass-ceramic. Dent Mater. 2016 Jul; 32(7):908-14. doi: 10.1016/j. dental.2016.03.013. Epub 2016 Apr 14.

16. Traini T, Sinjari B, Pascetta R, Serafini N, Perfetti G, Trisi P, Caputi S. The zirconia-reinforced lithium silicate ceramic: lights and shadows of a new material. Dent Mater J. 2016 Oct 1; 35(5):748-755. Epub 2016 Aug 20.

17. Paul. S, Peter. A and Pietrobon. N. Visual and spectrophotometric shade analysis of human teeth. J Dent Res, 2002; 81; 578-582.

18. Seghi. R, Hewlett. R, Kim. J. Visual and colorimetric assessment of small color differences on translucent dental porcelain. J Dent Res 1989; 68 (12): 1760-1764.

19. George A. Color theory and its application in art and design Mosby. 2nd edition 1987; 1-14.

20. Johnston WM. Color measurement in dentistry. J Dent 2009; 37 ; 2-6.

21. Paravina. D and Powers. M. Esthetic color training in dentistry. Saint Louis: Mosby; 2004; 43-4.

22. Wasson $\mathrm{W}$ and Schuman N. Color vision and dentistry. Quint Int 1992; 23:349-353.

23. Kuehni RG, Marcus RT. An experiment in visual scaling of small color differences Color Research and Application 1979; 28(6), 425-435.

24. Ruyter IE, K. Nilner, Moller B. Color stability of dental composite resin materials for crown and bridge veneers. Dent Mater 1987; 3:246-251.

25. Acar. O, Yilmaz. B, Altintas. SH, Chandrasekaran. I and Johnston. WM. Color stainability of CAD/CAM and nanocomposite resin materials. J Prosthet Dent. 2016 Jan; 115(1):71-5. 
26. Johnston WM, Kao EC. Assessment of appearance match by visual observation and clinical colorimetry J Dent Res 1989; 68(5):819-822

27. Yap. U, Tan. W, Tay. C, Chang. M, Loy. K and Mok. Y. Effect of mouth rinses on microhardness and wear of composite and compomer restoratives. Oper Dent 2003; 28: 740-6.

28. Anusavice KJ., "Recent Developments in Restorative Dental Ceramics". J Am Dent Assoc. 1993; 124(2):72-74, 76-78, 80-84.

29. Akbar JH, Petrie CS, Walker MP, Williams's k, Eick JD. Marginal adaptation of Cerec3 CAD/CAM composite crowns using two different finish line preparation designs. J Prosthodont 2006; 15:155-63.

30. Tysowsky G. Science behind lithium disilicate. Oral Health. March 2009:93-97.

31. Ingbert F., "The First Force Absorbing Flexible Nano Ceramic", J Prosthodont Res 2015.

32. Vichi A, DelSiena F, Sedda M, et al. Flexural resistance of CAD/CAM blocks for CEREC [Abstract 129]. J Dent Res. 2010.

33. Arocha. MA, Basilio J, Llopis J, Bella ED, Roig M, Ardu $\mathrm{S}$ and Mayoral JR. "Colour stainability of indirect CADCAM processed composites vs. conventionally laboratory processed composites after immersion in staining solutions. J Dent 2014 Jul; 42(7):831-8

34. Al- Wahab. Z. "An evaluation of the effect of different drinks on the color changes of Composan Ceram Composite (In Vitro Study)" MJD. 2009. Vol.: 6 No.:1

35. Abdesalam M. Effect of veneering technique and thickness on the color matching of translucent Ice zirconia substructure veneered with lithium disilicate using pressable technique versus conventional layering technique (in vitro study). Master thesis 2015.

36. El Sayed S M, Basheer R R, And Bahgat S F. Color Stability And Fracture Resistance Of Laminate Veneers Using Different Restorative Materials And Techniques. Egyptian Dental Journal April 2016; 62, 1-15.Studies IC.

37. Nakamura $T$, Wakabayashi $K$, Kinuta $S$, Nishida $H$, Miyamae M, yatani H. Mechanical propreties of new selfadhesive resin based cement. J Prosthodont Res. 2010; 54(2):59-64

38. Sarac D, Sarac YS, Yuzbasioglu E, Bal S. the effects of porcelain polishing systems on the color and surface texture of feldspathic porcelain. J Prosthet Dent 2006; 96:122-8.
39. Awliya. W, Al-Alwani. D, Gashmer. E and Al-Mandil. H. The effect of commonly used types of coffee on surface micro hardness and color stability of resin-based composite restorations. Saud Dent J 2010; 22:277-181 .

40. Cal E, Guneri P, Kose T. Comparison of digital and spectrophotometric measurements of color shade guides. J Oral Rehabil 2006; 33:221-8.

41. Samra A, Pereira S, Delgado L. and Borges C. Color stability evaluation of aesthetic restorative materials. Braz Oral Res. 2008 Jul-Sep; 22(3):205-10.

42. Al-Harbi,A;Ardu,S; Bortolotto, T; Krejci,I.: Stain intensity of CAD/CAM Materials versus Direct composites. IADR 2012 Poster Abstract, Iguaçu Falls, Brazil

43. Awad. D, Stawarczyk. B, Liebermann. A and Ilie. N. Translucency of esthetic dental restorative CAD/CAM materials and composite resins with respect to thickness and surface roughness. J Prosthet Dent. 2015 Jun; 113(6):534-40

44. Ataya. A, Karayazgan. B, Ozkan. Y, Akyil. MS. Effect of colored beverages on the color stability of feldspathic porcelain subjected to various surface treatments. Quintessence Int. 2009 Jul-Aug;40(7):e41-8

45. Ghahramanloo A, Madani AS, Sohrabi K, Sabzevari S. An evaluation of color stability of reinforced composite resin compared with dental porcelain in commonly consumed beverages. J Calif Dent Assoc 2008; 36(9):673-80.

46. Gawriolek M, Sikorska E, Ferreira LF, Costa AI Khmelinskii I, Krawczyk A, et al. Color and luminescence stability of selected dental materials in vitro. J Prosthodont 2012; 21:112-22.

47. Rosentritt M, Esch J, Behr M, Leibrock A, Handel J. In vivo color stability of resin composite veneers and acrylic resin teeth in removal partial dentures. Quintessence Int. 1998; 29(8):517-21 .

48. Um CM and Ruyter IE. Staining of resin-based veneering materials with coffee and tea. Quintessence Int. 1991; 22(5):377-86

49. Belli R, Geinzer E, Muschweck A, Petschelt A, Lohbauer U. Mechanical fatigue degradation of ceramics versus resin composites for dental restorations.Dent Mater 2014;30:424-32.

50. Vasudeva G. Monomer systems for dental composites and their future: a review. J Calif Dent Assoc 2009; 37:389-98. 
51. Gajewski VE, Pfeifer CS, Froes-Salgado NR, Boaro LC, Braga RR. Monomers used in resin composites: degree of conversion, mechanical properties and water sorption/ solubility. Braz Dent J 2012; 23:508-14.

52. Bagheri R, Burrow MF, Tyas M. Influence of foodsimulating solutions and surface finish on susceptibility to staining of aesthetic restorative materials. Journal of Dentistry 2005; 33:389-98.

53. Kugel, G. Direct and indirect adhesive restorative materials: a review. Am. J. Dent 2000; 13, 35-40.
54. Moszner, N., Fischer, U.K., Angermann, J., Rheinberger, V. A partially aromatic urethane dimethacrylate as a new substitute for Bis-GMA in restorative composites. Dent. Mater 2008; 24, 694-699.

55. Van Noort R. Introduction to dental materials. 2nd ed. St. Louis: Elsevier; 2007.p. 247-50.

56. Samra A, Ribeiro D, Borges C, Kossatz S. Influence of professional prophylaxis on reducing discoloration of different aesthetic restorative materials. Journal of dentistry 2012; 4 0s: e 71 -e7 6 